\title{
LA DÉRIVATION PROVISOIRE DU ZÊZZERE A CASTELO DO BODE (PORTUGAL)
}

\section{THE TEMPORARY DIVERSION OF THE ZEZERE RIVER} AT CASTELO DO BODE

par CARVALHO XEREZ

Ingénieur en Chef de l'Hidro-Eléctrica do Zêzere

English synopsis p. 563

Dans le cadre du plan d'équipement hydroélectrique du Portugal qui, sous l'impulsion donnée par le Gouvernement portugais, se trouve actuellement en cours de réalisation, on exécute présentement les travaux du Barrage et de la Centrale de Castelo do Bode, sur le Zêzere (affluent du Tage). Ces ouvrages font partie d'un ensemble destiné à utiliser, pour la production hydro-électrique, cette rivière qui fournira un total de 700 millions de Kwh annuels, dont 380 reviendront à la chute de Castelo do Bode.

Cette note décrira les travaux de dérivation provisoire du Zêzere, effectués selon les procédés courants pour les ouvrages de cette nature, mais la particularité, ici, réside dans le critère de sécurité qui a présidé à leur dimensionnement et dans le soin dont ont été entourées toutes les études, bien qu'il ne s'agisse pas d'ouvrages de caractère définitif.

Avant d'entrer dans le vif de cette note, il nous semble opportun de décrire sommairement le schéma d'aménagement du Zêzere et aussi les ouvrages de la chute de Castelo do Bode.

\section{L'aménagement du Zêzere.}

1. Le Zêzere, en raison de sa situation géographique, de la grande étendue de son bassin versant occupé en partie par une zone de précipitations élevées ef par suite d'autres conditions favorables, a été choisi pour constituer le grand système producteur d'énergie hydro-électrique du centre du pays.

C'est son cours inférieur qui présente les conditions les meilleures pour l'aménagement hydroélectrique, le schéma d'équipement établi tenant compte de la nécessité de créer des retenues de grande capacité pour régulariser un débit de régime très irrégulier et d'utiliser toute la chute disponible.

Comme il s'agit d'une rivière à faible pente, il a été facile de satisfaire simultanément à ces deux conditions car, en raison des particularités géologiques, on rencontre deux affleurements rocheux, un de granit à Cabril, et l'autre de schiste dur à Castelo do Bode, où il est possible de construire deux grands barrages dans de bonnes conditions économiques. Ces barrages, celui de Cabril d'une hauteur de $125 \mathrm{~m}$., et celui de Castelo do Bode d'une hauteur de $110 \mathrm{~m}$., créent des retenues d'une capacité utile de 500 et 875 millions de $\mathrm{m}^{3}$ et leurs centrales de pied de barrage, utilisent des chutes brutes moyennes de 95 et 80 mètres.

2. Pour utiliser la chute intermédiaire de $54 \mathrm{~m}$., on construira un barrage, dit de Bouçä, de $65 \mathrm{~m}$. de haut, sur un affleurement de granit situé à l'extrémité du remous de Castelo do Bode. On créera ainsi une retenue de 49 millions de $\mathrm{m}^{3}$ qui, sans jouer un rôle régularisateur appréciable, fournira toutefois une aide sensible pour les pointes quand les deux autres retenues atteindront leur niveau minimum. 
A l'aval de Castelo do Bode on prévoit de construire un barrage mobile avec centrale laté. rale créant un petit réservoir qui permettra une régularisation horaire des débits.

La fig. 1 représente le plan et le profil en long schématique de ce système et le tableau ci-dessous donne ses caractéristiques.
Le barrage est du type voûte épaisse de $115 \mathrm{~m}$. de hauteur maximum, avec un parement amont vertical de $150 \mathrm{~m}$. de rayon et un parement aval à inclinaison variable depuis 0,40 à la clé et augmentant vers les culées. Pour adapter au mieux la voûte aux formes topographiques de la vallée, on sera conduit à construire sur la rive droite

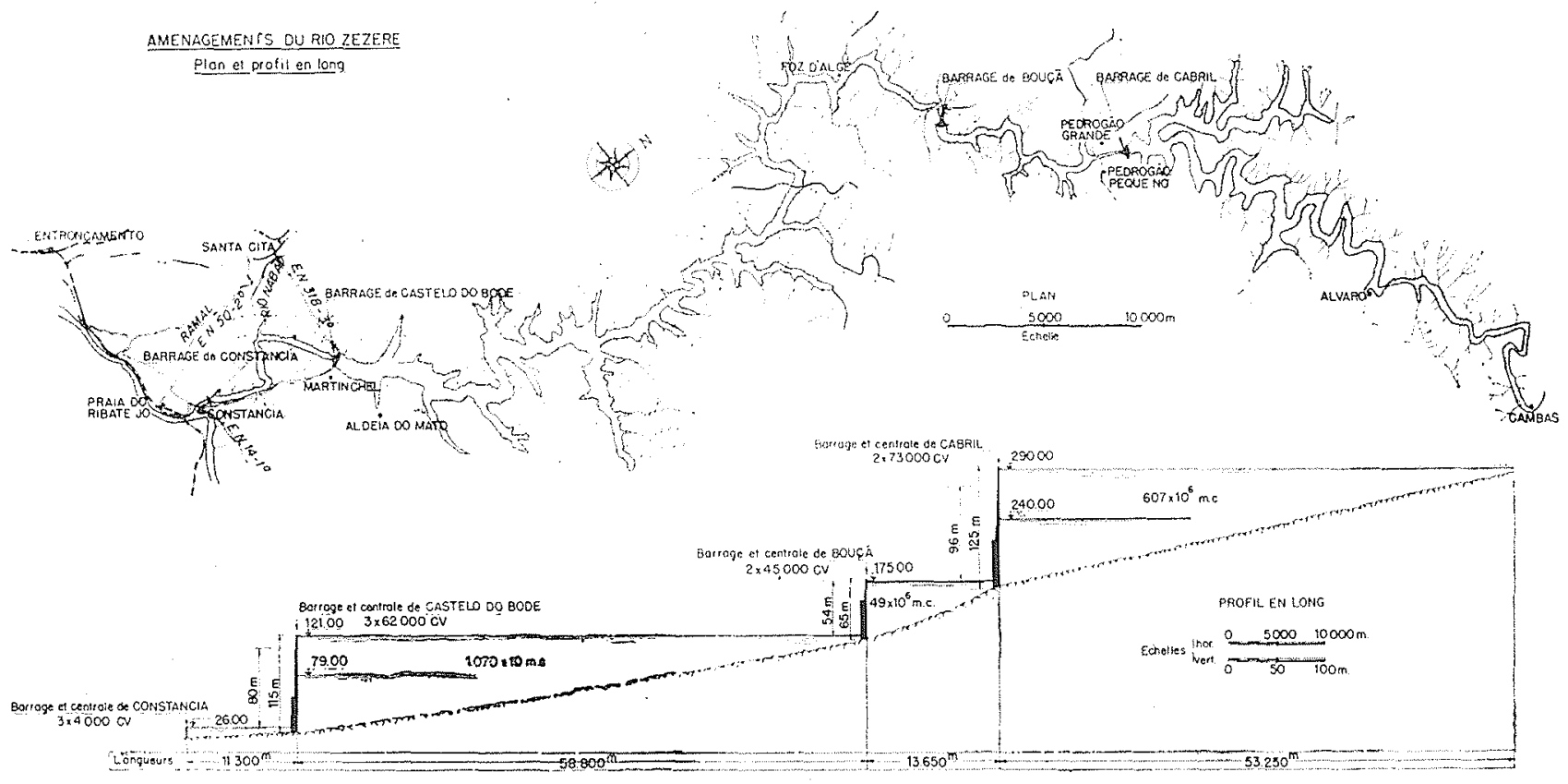

fig. 1

\begin{tabular}{|c|c|c|c|c|c|}
\hline $\begin{array}{c}\text { Situation des barrages } \\
\text { et des centrales } \\
\text { (pied de barrage) }\end{array}$ & $\begin{array}{l}\text { Hauteur } \\
\text { des barrages } \\
(\mathrm{m} .)\end{array}$ & $\begin{array}{l}\text { Capacité } \\
\text { totale } \\
\text { des retenues } \\
\text { (millions } \\
\text { de } \mathrm{m}^{3} \text { ) }\end{array}$ & $\begin{array}{l}\text { Chute brute } \\
(\mathrm{m} .)\end{array}$ & $\begin{array}{l}\text { Puissance } \\
\text { à installer } \\
\text { (CV) }\end{array}$ & $\begin{array}{l}\text { Energie } \\
\text { permanente } \\
\text { annuelle } \\
\text { (millions } \\
\text { de Kwh) }\end{array}$ \\
\hline Cabril & 125 & 600 & 115 à 65 & 146.000 & 190 \\
\hline Bouça & 65 & 49 & 54 & 90.000 & 110 \\
\hline Castelo do Bode & 115 & 1.070 & 95 à 53 & 186.000 & 380 \\
\hline \multirow[t]{2}{*}{ Constância } & 12 & 5 & 7 & 12.000 & 20 \\
\hline & & & Totaux & 434.000 & 700 \\
\hline
\end{tabular}

\section{La chure de Castelo do Bode.}

3. Les ouvrages définitifs de Castelo do Bode (fig. 2) constitués par les trois organes classiques - barrage, centrale et évacuateur de crues ont été étudiés en vue de concentrer ces organes pour faciliter leur construction, sans qu'ils soient toutefois interdépendants, ce qui ferait perdre l'effet de simplification désiré. une culée d'une certaine importance. Le volume de béton à mettre en place est de $400.000 \mathrm{~m}^{3}$ et celui des excavations pour les fondations de $100.000 \mathrm{~m}^{3}$.

4. L'évacuateur de crues établi pour un débit de $4.000 \mathrm{~m}^{3} / \mathrm{sec}$. est situé sur le barrage près de la rive gauche, complètement indépendant de la centrale. On a effectué une étude très 
Septembre-Octobre 1949

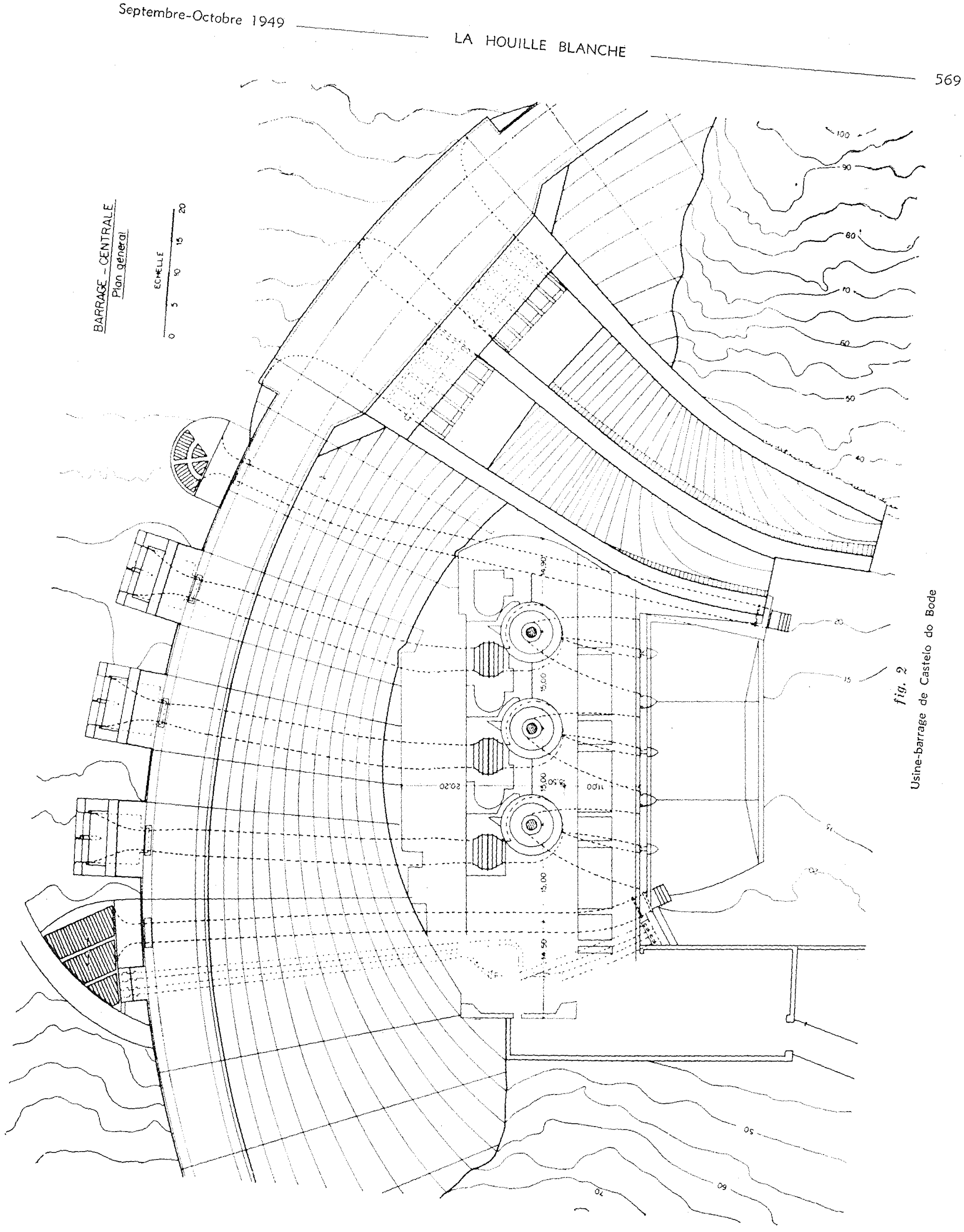




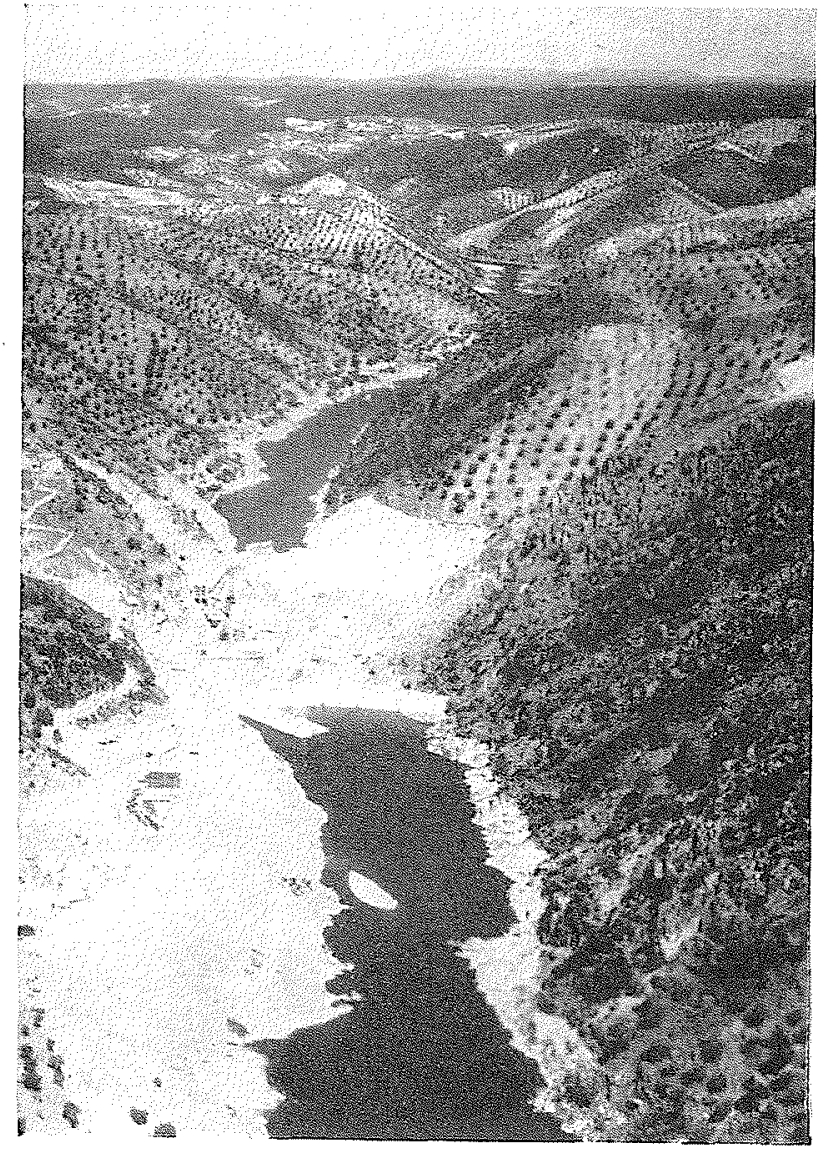

fig. 3

Le chantier du barrage ef le Rio Zêzere, vus d'avion

complète en modèle réduit, étude qui a porté principalement sur l'entonnement et la restitution des crues.

Pour l'entonnement, on a cherché une solution qui permette de faire couler l'eau sur le parement aval sans surépaisseur de béton. II n'était pas possible, dans ces conditions, de prévoir un profil Creager avec écoulement à surface libre, et par suite on a adopté un artifice, à savoir. établir un écoulement en charge à travers deux orifices, le débit étant réglé par des vannes secteur montées à leur sortie (cf. fig. 4).

L'étude de la restitution de l'eau à l'aval a présenté des difficultés particulières: on devait lutter contre le peu d'espace disponible entre la centrale et le terrain, et en outre il fallait lancer l'eau sensiblement dans l'axe de la rivière pour éviter d'attaquer les rives. La première difficulté a été résolue en déployant les jets en hauteur, ce qui présente en plus l'avantage de mieux disperser l'énergie dans l'air. A la suite de nombreux essais portant sur les formes de la partie aval de l'évacuateur, on a réussi à lancer parfaitement l'eau dans l'axe de la rivière et on a obtenu fina. lement une solution comportant des jets rasants, pour laquelle il se forme une fosse stable, sans aucun phénomène d'érosion régressive susceptible de compromettre la stabilité des ouvrages.

5. Dans la centrale seront installés trois groupes turbo-alternateurs à axe vertical d'une puissance de $62.000 \mathrm{CV}$, sous la chute moyenne de $80 \mathrm{~m}$., protégés à l'amont par des vannes sphériques de $3 \mathrm{~m}$. 30 de diamètre. Les trois conduites (fig. 5), de $4 \mathrm{~m}$. de diamètre, qui alimentent les groupes, traversent le barrage; leurs parois ont une épaisseur inférieure à celle qui serait nécessaire si elles étaient à l'air libre, et sont munies de cornières de renfort contre les pressions extérieures provoquées par les infiltrations éventuelles dans le barrage. L'ensemble est protégé à l'amont par des vannes à chenilles assurant la fermeture rapide en cas d'accident.

Les alternateurs sont établis pour une puissance continue maximum de 57.400 KVA sous 15.000 Volts. Leur ventilation s'effectue en circuit fermé. Les transformateurs 15/150 KV., couplage étoile-triangle, sont installés à l'aval de la salle des machines. Les lignes partent alors en direction de la sous-station à construire sur la rive droite, en prenant appui sur la façade du bâtiment.

Les vidanges de fond, au nombre de deux, traversent le barrage et la centrale, de part et d'autre des groupes. Elles sont constituées par des conduites de $3 \mathrm{~m}$. de diamètre et des vannes cylindriques équilibrées, le tout protégé à l'amont par des vannes à chenilles.

\section{Les ouvrages de dérivation provisoire (fig, $6,7,8$ )}

6. C'est le critère déjà mentionné qu'on a suivi pour établir le projet des ouvrages de dérivation provisoire: on les a considérés comme un facteur d'importance fondamentale dans l'ensemble des travaux à effectuer, et ceci en raison de leur influence très marquée sur les délais d'exécution des ouvrages définitifs.

S'il est nécessaire, d'une part, de protéger le chantier contre un débit de crues suffisant, pour réduire les risques d'inondation et ne pas retarder l'avancement des travaux, il faut, d'autre part. que les ouvrages de dérivation soient d'exécution rapide et facile, afin que le délai de construction total soit le plus court possible. C'est ce juste 


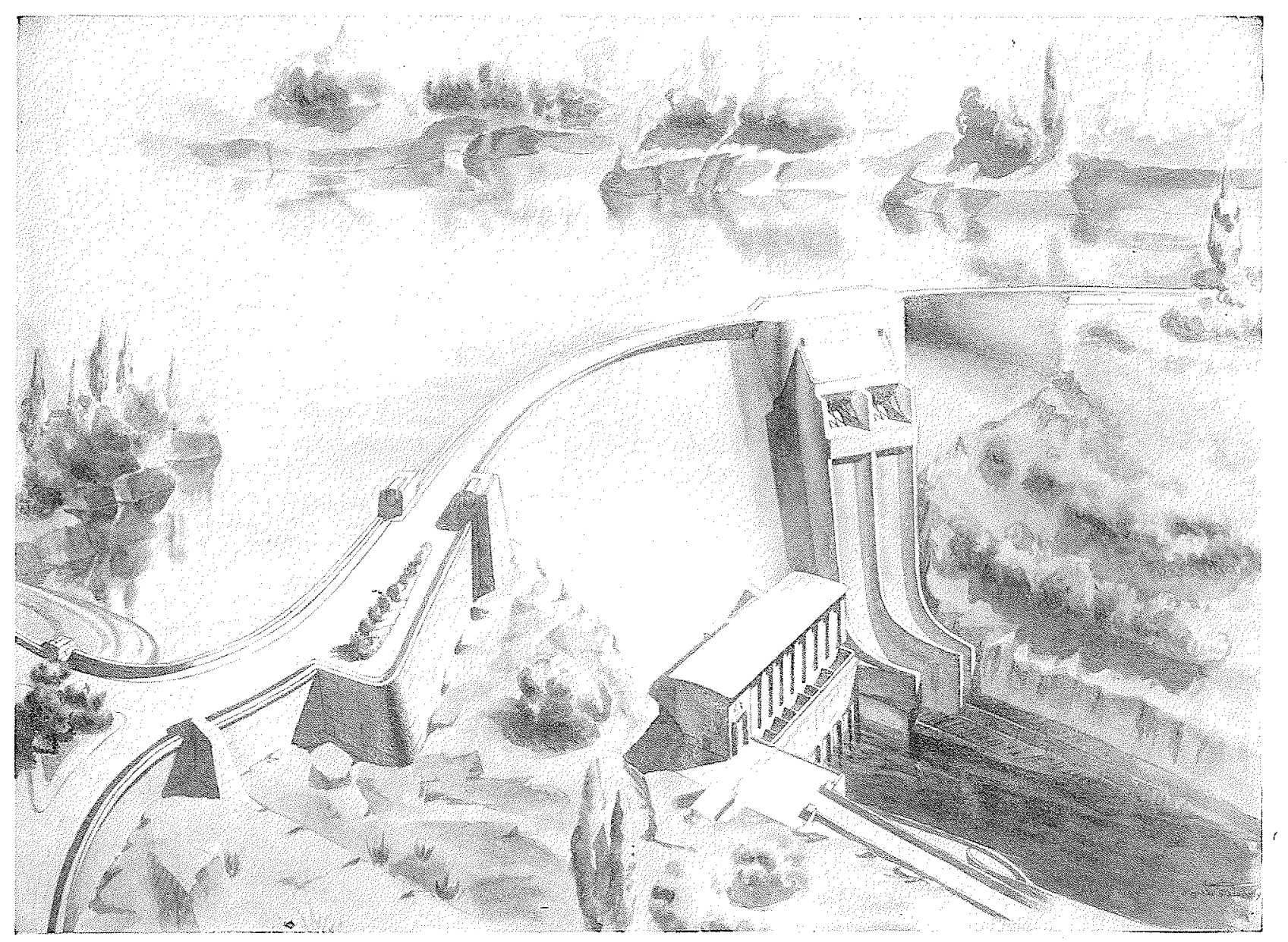

fig. 4

Barrage-usine de Castelo do Bode: maquette des ouvrages (remarquer l'évacuateur de crues)

équilibre entre ces deux conditions et le coût des ouvrages qui caractérise la solution optimum.

Ces considérations appliquées de façon plus ou moins parfaite au cas présent ont conduit en premier lieu à fixer à $2.000 \mathrm{~m}^{3} / \mathrm{sec}$. le débit dérivable, ce qui correspond à une protection du chantier contre une pointe de crue d'environ $2.700 \mathrm{~m}^{3} / \mathrm{sec}$, compte tenu de l'effet régularisateur de la retenue créé par le batardeau amont. Ensuite, pour satisfaire aux conditions de rapidité et d'économie des travaux, on a adopté, comme solution, une galerie unique sur la rive droite, fonctionnant en charge à une vitesse de l'ordre de $21 \mathrm{~m} . / \mathrm{sec}$. pour le débit maximum.

L'avantage de ce mode de fonctionnement est qu'il conduit à des vitesses variant avec le débit, ce qui permet d'accepter la vitesse maximum indiquée. Elle ne se produira en effet qu'excep- tionnellement, puisque, pour les débits ordinairement dérivés, les vitesses sont très inférieures à la vitesse maximum. En acceptant cette vitesse maximum, on a obtenu une section de galerie aussi faible que possible par rapport à la valeur du débit à dériver; la section est définie par des arcs de cercles; sa largeur est de $12 \mathrm{~m}$. et sa hauteur de $10 \mathrm{~m}$.

Il est facile d'obtenir une bonne récupération d'énergie cinétique à l'aval de la galerie, au moyen de dispositions adéquates de la tête aval, judicieusement étudiées sur modèle réduit. On peut ainsi opter pour une galerie calée aussi bas que le permettent les conditions de récupération, ce qui donne la hauteur minimum pour le batardeau amont, ou bien, si d'autres considérations le justifient, caler la galerie légèrement plus haut, la cote du batardeau amont augmentant alors de la même quantité. 


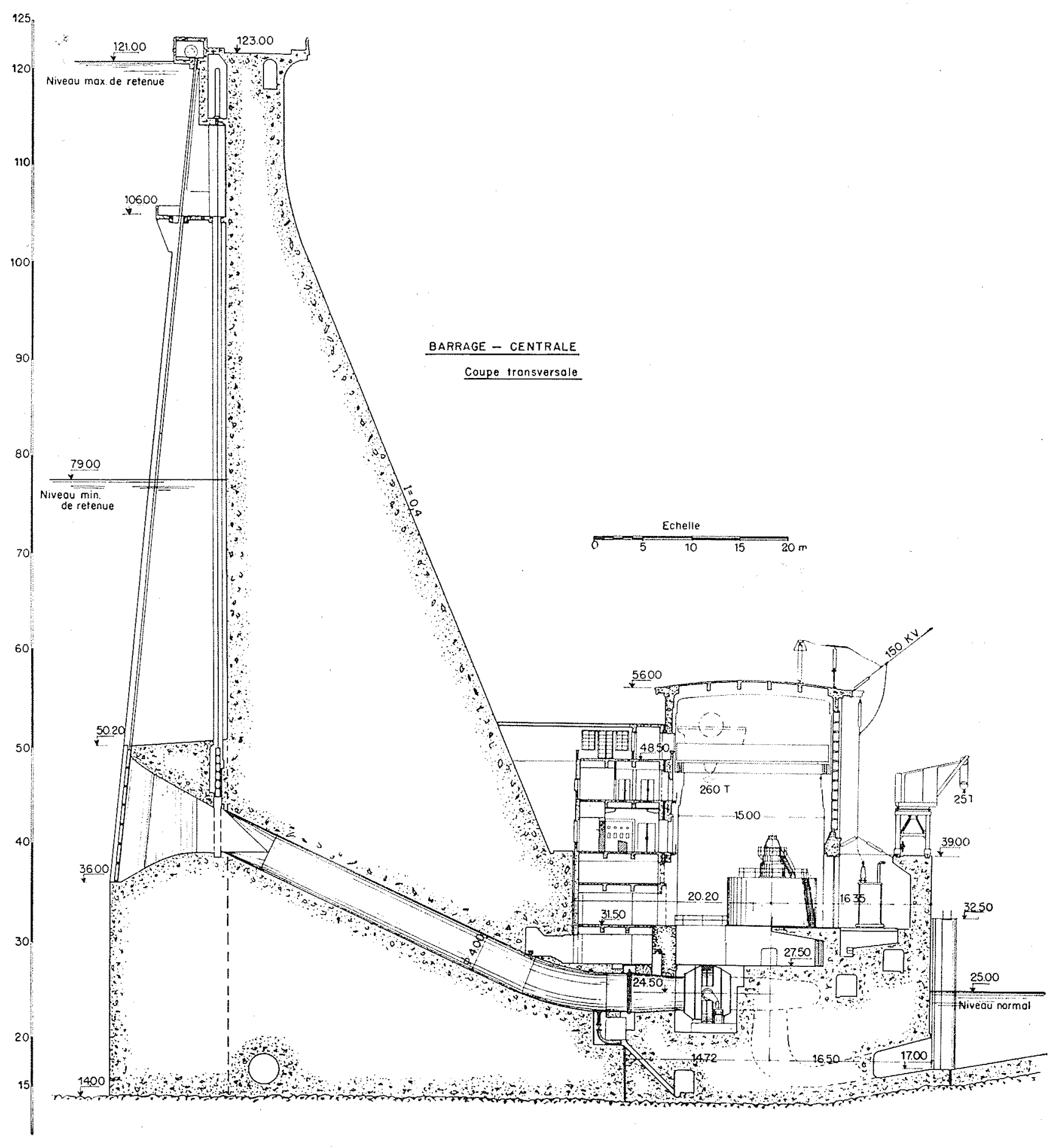

fig. 5

L'usine-barrage de Castelo do Bode 


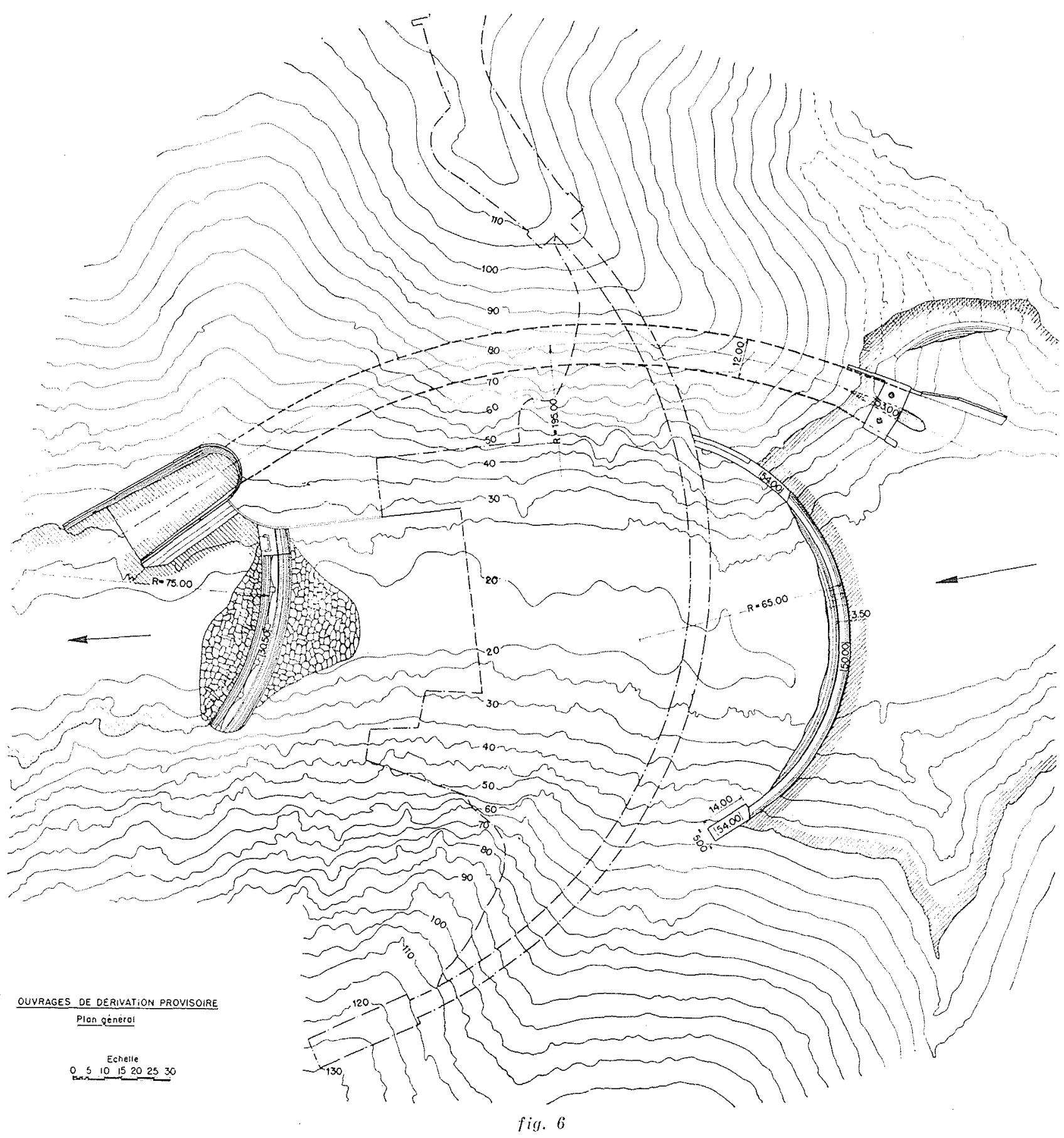

Le chantier de I'usine-barrage de Castelo do Bode

Dans ces conditions, on a commencé par fixer à $24,20 \mathrm{~m}$. le niveau du point le plus bas du plafond de la galerie, de façon à permettre le passage des troncs d'arbres flottés pendant l'étiage; de cette cote résulte la valeur de $(50,00 \mathrm{~m}$.) pour la cote du couronnement du batardeau amont. La pente du fond de la galerie a été fixée, pour des raisons constructives, à $1 \mathrm{~cm} / \mathrm{m}$.
7. Toutes ces données, dont nous venons de donner les justifications, ont servi de base pour la construction d'un modèle réduit à l'échelle de $1 / 50^{\circ}$ destiné à étudier les formes des têtes amont et aval (1).

(1) Dans un très prochain numéro, nous publierons un article relatif à ces essais sur modèle réduit. 
Pour la tête amont, les études ont porté sur les formes du radier, du masque de mise en charge, des murs guideaux et du pilier central destiné à diviser la section en deux passes pour faciliter son obturation par des vannes

Les essais de la tête aval furent plus délicats parce que l'on cherchait les formes du radier et des murs latéraux qui permettent d'obtenir la meilleure récupération d'énergie possible. Le fonctionnement hydraulique de la tête aval est le suivant: elle reste noyée jusqu'à $1.400 \mathrm{~m}^{3} / \mathrm{sec}$. environ - un ressaut ondulé se formant jusqu'à $800 \mathrm{~m}^{3} / \mathrm{sec}$. et à mesure que le débit augmente il se produit une certaine turbulence, très accentuée vers $1.200 \mathrm{~m}^{3} / \mathrm{sec}$. - après quoi la tête se dénoie complètement en chassant le ressaut vers l'aval. La mise en charge de la galerie s'effectue par l'aval, et remonte progressivement jusqu'à la tête amont qui se trouve complètement en charge pour un débit de $1.000 \mathrm{~m}^{3} / \mathrm{sec}$.
8. Les travaux ont été commencés, en avril 1946, par la construction de la galerie. La méthode de travail a consisté à ouvrir deux

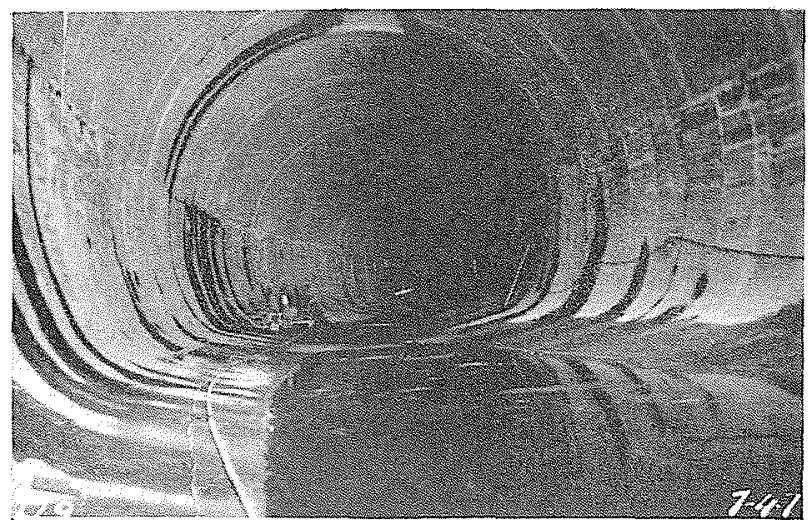

fig. 7

La galerie de derivation

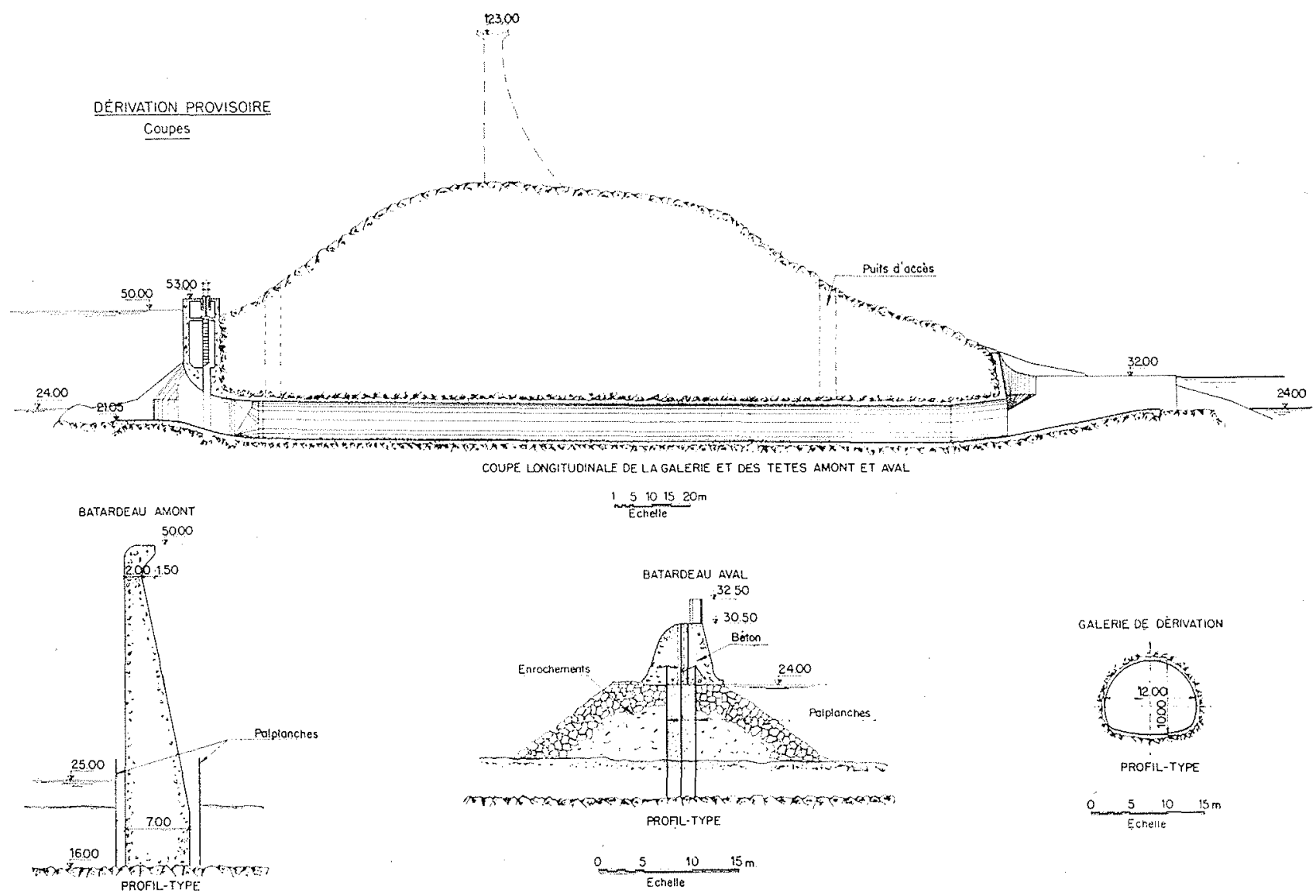

fig. 8

Usine-barrage de Castelo do Bode. - Les ouvrages de dérivation provisoire : batardeaux et galerie 
puits (fig. 8) à une distance de chacune des têtes telle que leur entrée débouche au-dessus du niveau des plus grandes crues, ce qui a permis de travailler à 4 fronts durant toute l'année. Les produits d'excavation ont été remontés par ces puits avec deux petites grues électriques.

Le creusement et le revêtement ont été réalisés simultanément non pas parce que la nature de la roche l'exigeait, mais surtout dans le but d'accélérer le rythme des travaux et de protéger le personnel contre les chutes de pierres; on a ainsi adopté la méthode générale suivante: après avoir effectué le forage de la galerie d'avancement classique à la partie supérieure, on a creusé la moitié supérieure de la galerie et l'on a exécuté le revêtement correspondant d'une épaisseur moyenne de $50 \mathrm{~cm}$. On a continué par l'excavation de la moitié inférieure en laissant



fig. 9

Construction de la tête aval de la dérivation

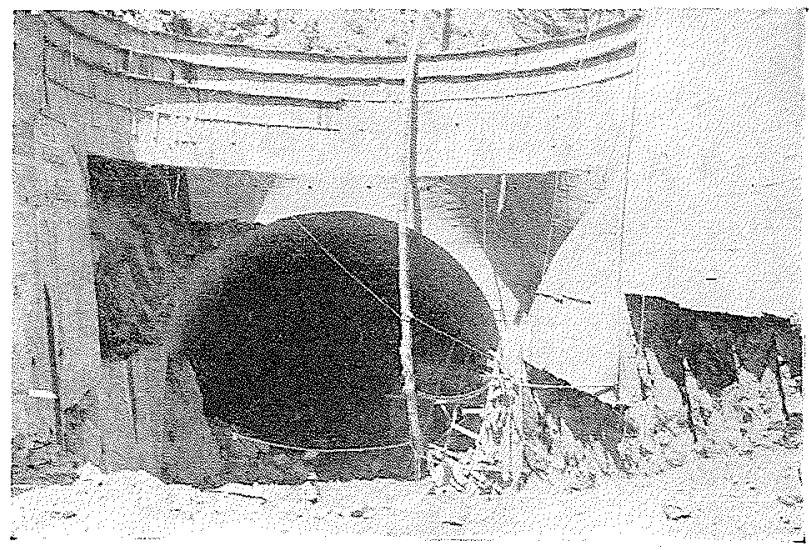

fig. 10

Tête aval de la dérivation

toutefois subsister des massifs latéraux pour servir d'appui au revêtement supérieur; le reste du revêtement, de la même épaisseur, a été commencé par le radier et finalement on a exécuté les piédroits par tronçons alternés de $5 \mathrm{~m}$., de façon à garantir la stabilité de la voûte.

L'excavation et le chargement des déblais ont été entièrement effectués par des moyens manuels, en utilisant des marteaux pneumatiques légers, ce qui, étant donné l'excellente qualité de la main-d'œuvre portugaise, a permis d'obtenir de bons rendements de travail. Les coffrages ont été construits en bois, se déplaçant sur rail, et le béton a été mis en place au moyen d'une pompe de $15 \mathrm{~m}^{3}$ /heure.

9. Les têtes amont et aval, outre le grand volume d'excavation qu'elles nécessitaient, comprenaient des ouvrages importants de béton

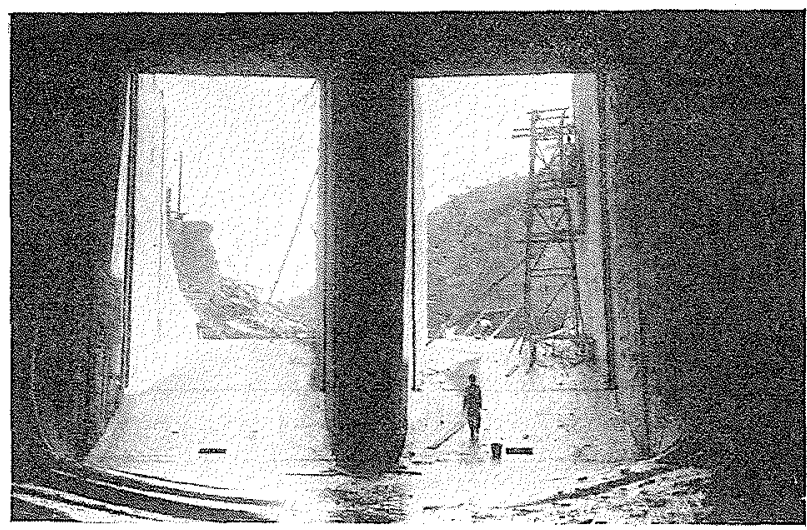

fig. 11

Construction de la tête amont de la dérivation (photo prise de l'intérieur de la galerie) 


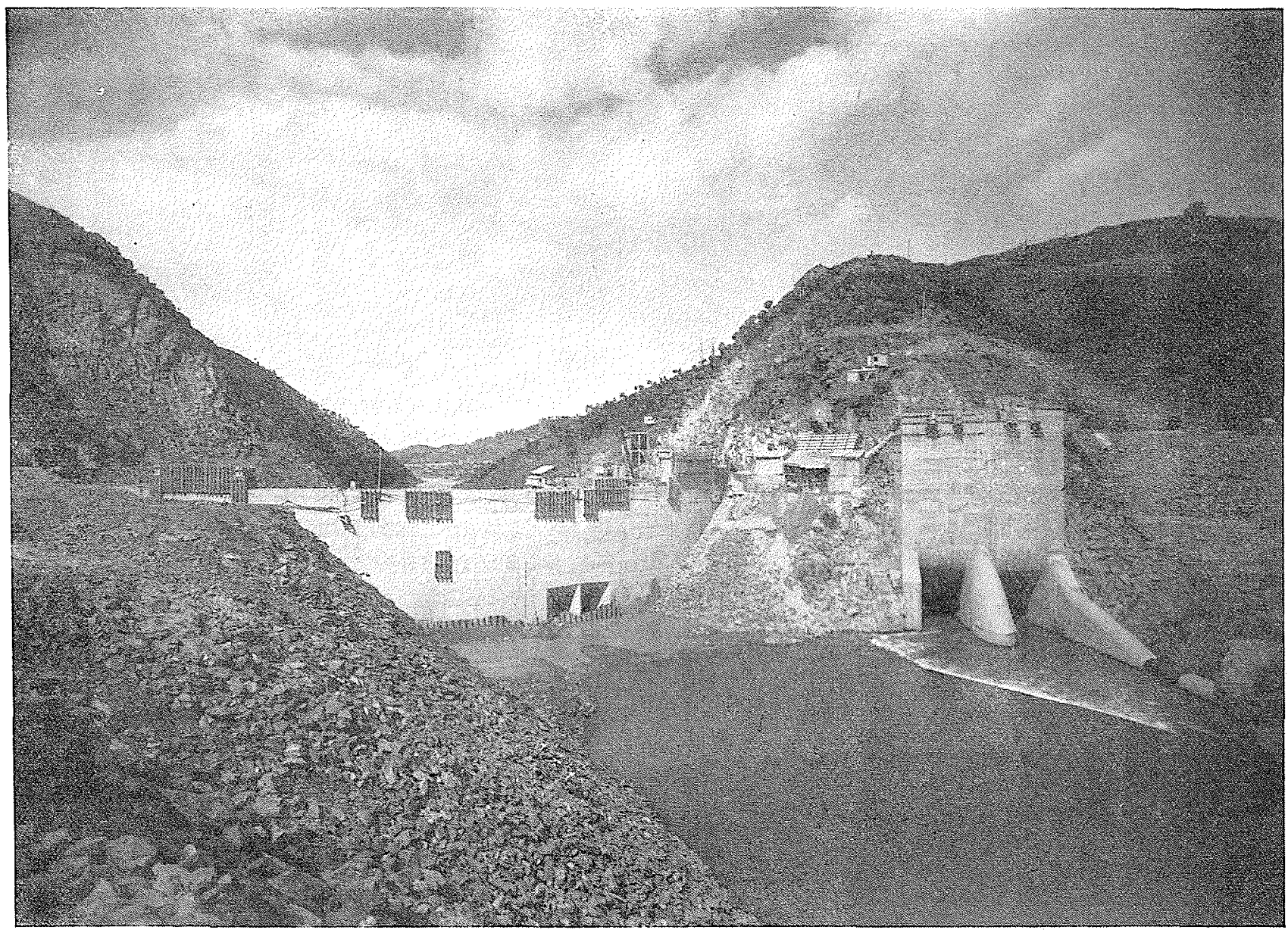

fig. 12

Batardeau amont ef tête amont de la dérivation

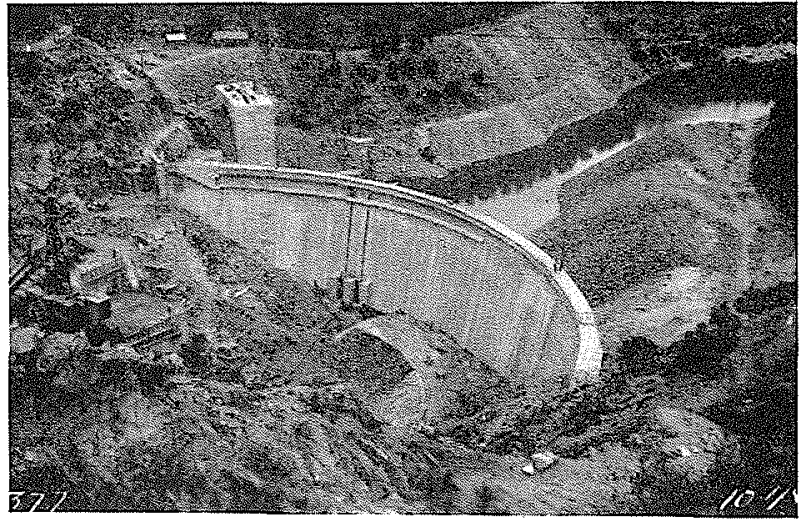

fig. 13

Le batardeau amont

armé, particulièrement à l'amont, où il a fallu construire une chambre destinée à abriter les deux vannes Stoney, qui devront fermer la galerie au moment où l'on construira son bouchon, ainsi que les organes de manœuvre de ces vannes.

L'exécution de ces têtes a été menée de pair avec celle de la galerie et l'on a dû construire deux batardeaux-voûtes minces pour protéger les travaux, afin de pouvoir les continuer durant l'hiver 1946-47.

Tout le radier et les murs de revêtement ont été soigneusement ancrés au rocher, afin de garantir une protection efficace contre les souspressions dans le premier cas et une stabilité convenable au passage de l'eau à grandes vitesses dans le second.

Les excavations et le bétonnage ont été effectués à l'aide de grues électriques et de la pompe à béton déjà mentionnée.

10. Le batardeau amont est un barragevoûte mince de $35 \mathrm{~m}$. de hauteur maximum, 

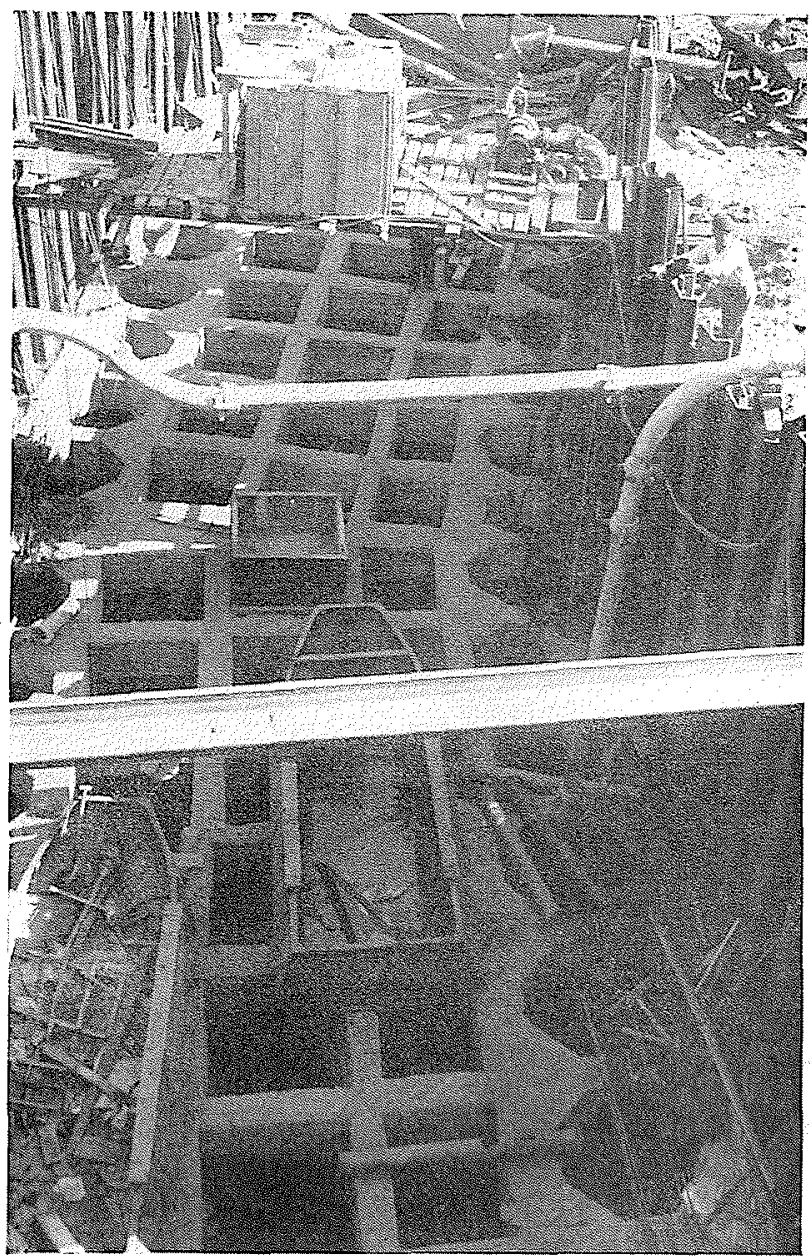

fig. 1/.

Le contreventement entre rideaux de palplanches lors de l'édification du batardeau amont

fondé sur le rocher et de développement en crête égal à $115 \mathrm{~m}$. Sa forme est définie ainsi :

- parement amont vertical de $65 \mathrm{~m}$. de rayon ;

- parement aval avec fruit de 0.20 ;

- épaisseur de $7 \mathrm{~m}$. à la base et épaisseur minimum de $2 \mathrm{~m}$. au couronne. ment.

Le couronnement est muni d'une crête déversante, afin de permettre l'évacuation des crues au cas où leur valeur excéderait le débit de $2.000 \mathrm{~m}^{3} / \mathrm{sec}$. que la galerie peut dériver.

Le batardeau est équipé de deux vannes-wagon disposées sur le parement aval et obturant une section totale de $8 \mathrm{~m}^{2}$ qui permet de débiter $50 \mathrm{~m}^{3} / \mathrm{sec}$. sous charge réduite. Il serait ainsi possible de faire passer par ces vannes le débit de la rivière dans une période d'eaux moyennes si l'on désirait effectuer une réparation dans la galerie ou bien pour construire le bouchon qui l'obturera définitivement.

On a construit le batardeau par blocs indépendants, séparés par les joints de contraction classiques tous les 12 mètres environ, munis de lames de cuivre à l'amont et de dispositifs d'injection.

La partie de cet ouvrage en dessous du niveau d'étiage, c'est-à-dire en dessous de la cote $25 \mathrm{~m}$., a été construite selon un procédé spécial. On avait en effet constaté que le battage de palplanches à travers les alluvions, jusqu'à atteindre le rocher en place, s'effectuait avec une grande facilité et que l'on pouvait, de cette façon, constituer aisément des cellules étanches.

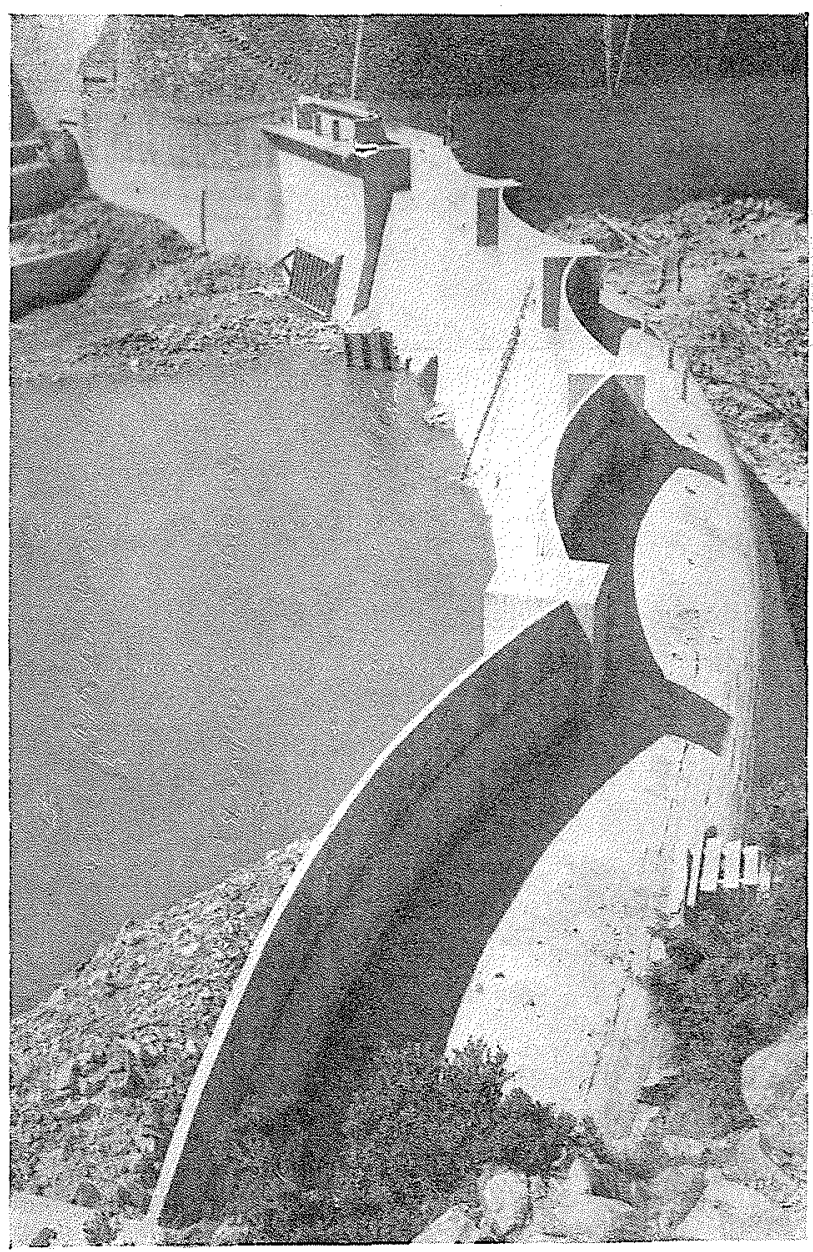

fig. 15

Le batardeau aval vu de la rive gauche 




fig. 16

Chute de Castelo do Bode: le chantier vu d'amont et de la rive gauche

On a ainsi décidé de battre deux rideaux de palplanches distants de $8 \mathrm{~m}$. (soit un mètre de plus que la largeur du batardeau). Ces rideaux suivent sensiblement le tracé du batardeau et sont divisés en cellules par des voiles transversaux. On a fait une première expérience en 1946. contre la rive droite, avec des résultats si satisfaisants qu'ils nous ont poussé à préparer deux portières flottantes sur lesquelles on a monté un derrick muni d'un trépideur et une sonnette; la mobilité de ce dispositif a permis une vitesse de battage telle que les deux rideaux ont pu être exécutés entre les mois d'avril et d'août 1947 , époque à laquelle on a commencé à dériver les eaux par la galerie.

Le contreventement entre les rideaux a été réalisé d'une manière originale au moyen de cadres horizontaux de béton armé qui ont été incorporés dans le corps du batardeau.
Au fur et à mesure que progressait le battage des rideaux et que l'on fermait et contreventait les cellules, on extrayait les alluvions. Une fois mis à nu le rocher, qui s'est toujours révélé sain, on coulait le béton entre des coffrages en bois plaqués contre les palplanches. Le bétonnage des blocs s'est poursuivi à un rythme accéléré de sorte que, en septembre 1947 , tous les blocs avaient atteint la cote $26 \mathrm{~m}$., ce qui permettait d'épuiser l'eau entre les deux batardeaux; on avait ainsi accompli le programme de travaux prévu. La mise en place du béton, pour les blocs voisins de la rive droite, a été effectuée au moyen de wagonnets et plus tard on a utilisé un blondin de deux tonnes.

La progression des travaux a permis d'atteindre, à la fin de 1947 , le niveau $(43 \mathrm{~m} .00)$ et de cette façon le chantier s'est trouvé protégé contre une crue de $1.600 \mathrm{~m}^{3} / \mathrm{sec}$. au cours de 
l'hiver 1947-48. Le niveau du couronnement a été atteint dans le courant de 1948. Les injections de ciment dans les joints ont été effectuées en deux phases correspondant aux deux phases de construction ci-dessus mentionnées.

11. Le batardeau aval est en enrochements, avec noyau imperméable constitué par un double rideau de palplanches battues dans les alluvions. Il est couronné par un massif en béton légèrement armé. assurant la stabilité du batardeau lors d'un déversement, en cas d'inondation du chantier. Les derniers mètres, pour atteindre la cote maximum $32,50 \mathrm{~m}$., sont constitués par une série de voûtelettes en béton légèrement armé et présentant leur concavité vers l'amont, de façon à ce que la pression de l'eau, si le chantier venait à être inondé par l'amont, les détruise automatiquement. On abaisserait ainsi le niveau de l'eau dans le chantier, ce qui permettrait d'éviter des dégats importants.

Le batardeau aval, comme le batardeau amont, est équipé d'une vidange prévue pour $50 \mathrm{~m}^{3} / \mathrm{sec}$. et munie d'un clapet qui s'ouvrira sous l'effet de la pression en cas d'inondation du chantier par l'amont, permettant ainsi d'égaliser les niveaux à l'amont et à l'aval.

Les palplanches ont été battues au moyen de l'équipement flottant déjà mentionné, avec une certaine facilité. Ce travail était pratiquement terminé fin septembre 1947, ce qui a permis de mettre à sec le chantier ainsi que nous l'avons déjà signalé. Ce n'est que fin octobre que l'on a terminé les travalix de mise en place des enrochements et du béton.

12. En terminant cette note sur les ouvrages de dérivation provisoire, nous ne voudrions pas laisser de mentionner que les ouvrages définitifs - barrage et centrale - s'exécutent à un rythme accéléré grâce à la forte puissance de l'équipement de chantier que le maitre de l'œuvre a mis à la disposition de l'entrepreneur, et l'on espère que les travaux seront terminés fin 1950, date à laquelle entreront en service les deux premiers groupes.

Nous donnerons d'ici peu, dans cette revue, une description de l'exécution des travaux définitifs.

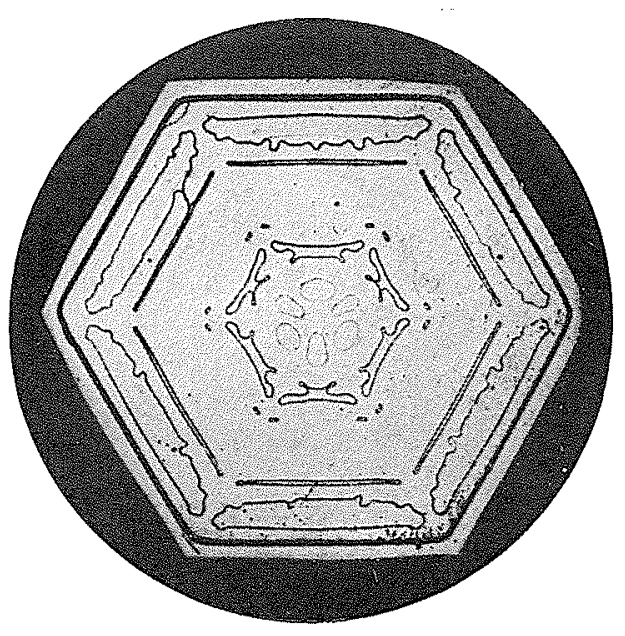

